\title{
Residual gas entering high density hydrogen plasma: rarefaction due to rapid heating
}

N. Den Harder, ${ }^{1}$ D. C. Schram, ${ }^{1}$ W. J. Goedheer, ${ }^{1}$ H. J. De Blank, ${ }^{1}$ M. C. M. Van de Sanden, ${ }^{1,2}$ and G. J. Van Rooij ${ }^{1}$

1) FOM Institute DIFFER - Dutch Institute for Fundamental Energy Research, Association EURATOM-FOM, Trilateral Euregio Cluster, Nieuwegein, The Netherlands

2) Department of Applied Physics, Eindhoven University of Technology, PO Box 513, 5600 MB Eindhoven, The Netherlands

(Dated: 30 November 2015)

Interaction of background molecular hydrogen with magnetized (0.4 T) high density $\left(1-5 \times 10^{20} \mathrm{~m}^{-3}\right)$ low temperature $(\sim 3 \mathrm{eV})$ hydrogen plasma was inferred from the Fulcher band emission in the linear plasma generator Pilot-PSI. In the plasma centre, vibrational temperatures reached $1 \mathrm{eV}$. Rotational temperatures obtained from the $\mathrm{Q}(v=1)$-branch were systematically $\sim 0.1 \mathrm{eV}$ lower than the $\mathrm{Q}(v=0)$-branch temperatures, which were in the range of $0.4-0.8 \mathrm{eV}$, typically $60 \%$ of the translational temperature (determined from the width of the same spectral lines). The latter is attributed to preferential excitation of translational degrees of freedom in collisions with ions on the timescale of their in-plasma residence time. Doppler shifts revealed co-rotation of the molecules with the plasma at an order of magnitude lower angular velocity, confirming that the Fulcher emission connects to background molecules. A simple model estimated a factor of 90 rarefaction of the molecular density at the centre of the plasma column compared to the residual gas density. Temperature and density information was combined to conclude that ion-conversion molecular assisted recombination dominates plasma recombination at a rate of $1 \times 10^{-15} \mathrm{~m}^{3} \mathrm{~s}^{-1}$. The observations illustrate the general significance of rapid molecule heating in high density hydrogen plasma for estimating molecular processes and how this affects Fulcher spectroscopy. 


\section{INTRODUCTION}

Low temperature hydrogen plasma is, amongst others, characterized by the significant presence of $\mathrm{H}_{2}$ molecules. The significance lies not so much in their relative abundance, but merely in their importance for opening up reaction pathways for the plasma ions. As a consequence, the properties of these molecules often determine main system properties such as plasma recombination (of interest for e.g. in the exhaust of fusion devices ${ }^{1,2}$ ), ionization and dissociation rates, and negative ion formation efficacy (both of interest for reactive species generation for materials processing in semiconductor industry ${ }^{3-5}$ ).

The present research in the linear plasma generator Pilot-PSI ${ }^{6}$ was conducted to reveal exactly one of such a system property, namely the plasma recombination rate, and in addition, to identify the exact molecular process responsible for plasma recombination. The various molecular plasma loss mechanisms, termed "Molecular Activated Recombination" (MAR) were suspected to be relevant on basis of previous work ${ }^{7,8}$. Out of the different MAR mechanisms, negative-ion-mediated MAR

$$
\begin{gathered}
e+H_{2} \rightarrow\left(H_{2}^{-}\right)^{*} \rightarrow H+H^{-} \\
H^{+}+H^{-} \rightarrow H+H(n=3)
\end{gathered}
$$

and ion-conversion MAR

$$
\begin{gathered}
H^{+}+H_{2} \rightarrow H+H_{2}^{+} \\
e+H_{2}^{+}\left(v^{\prime}\right) \rightarrow H(1 s)+H(n \geq 2)
\end{gathered}
$$

are the most important ${ }^{9}$. The rate of both recombination reactions is not only determined by the molecular density, but also strongly dependent on the rovibrational excitation of the reactants. An increase in the rovibrational energy of the molecules speeds up the reaction, whilst the reaction rate is enhanced by many orders of magnitude for the higher lying vibrational levels.

A wide range of active laser spectroscopic techniques is capable of measuring (vibrationally excited) hydrogen molecules, such as the LIF (Laser-Induced Fluorescence) ${ }^{10}$, CARS (Coherent Anti-Stokes Raman Spectroscopy) ${ }^{11}$, REMPI (Resonance-Enhanced MultiPhoton Ionization) ${ }^{12}$ and FWFM (Four-Wave Frequency Mixing) ${ }^{13}$. We chose for a passive spectroscopy approach that gives immediate overview over (parts of) the relative distribution over vibrational levels and that is commonly used in laboratory as well as fusion 
experiments $^{14-18}$. In particular, we studied the molecular emission of the diagonal Fulcher- $\alpha$ band $^{19}\left(d^{3} \Pi_{u} \rightarrow a^{3} \Sigma_{g}^{+}\right)$to determine vibrational temperatures ${ }^{20}$. The vibrational temperature was subsequently used to calculate the effective rate for both recombination reactions invoking available vibrationally resolved rate constants ${ }^{21}$. Similarly, the rotational temperature of the excited state ${ }^{22}$ was determined and, with assumptions about the excitation mechanism, projected to the ground state rotational temperature. The latter is often interpreted as the gas temperature. We determined the gas temperature independently from the Doppler broadening of the spectral lines and compared the two results. Doppler shifts were used to quantify the rotational motion and to assess the coupling between molecules and plasma ions. Finally, the molecular density in the plasma centre was inferred from these temperature measurements, enabling estimation of the absolute importance of the main MAR mechanisms.

The here reported experimental observations represent a general example of how fast molecule heating in high density hydrogen plasma affects the reaction rates of molecular processes and their overall importance. Furthermore, it illustrates how it affects the interpretation of Fulcher spectroscopy. These findings are not only of relevance for laboratory experiments, but also for future fusion devices such as ITER, in which the plasma conditions in the regions of the strike points at the divertor target are close to those in the present study ${ }^{23,24}$.

\section{EXPERIMENTAL}

\section{A. Instrumentation}

The linear plasma generator Pilot-PSI is used to generate hydrogen plasma beams of typically $12 \mathrm{~mm}$ wide (density FWHM), with a peak density and temperature of $1-5 \times$ $10^{20} \mathrm{~m}^{-3}$ and $1-3 \mathrm{eV}$, in a magnetic field of $0.4 \mathrm{~T}$. At these densities and temperatures, the collisionality is high, so that the electron and ion energy distributions are close to Maxwellian. Lifetimes of $\mathrm{H}_{2}^{+}$and $\mathrm{H}_{3}^{+}$ions are short, and protons are the majority ions. The ionization degree inside the plasma beam is high, more than $80 \%$ in the conditions used in this paper ${ }^{25}$.

A schematic of the device is shown in Figure 1. The plasma source is a cascaded $\operatorname{arc}^{26}$, 


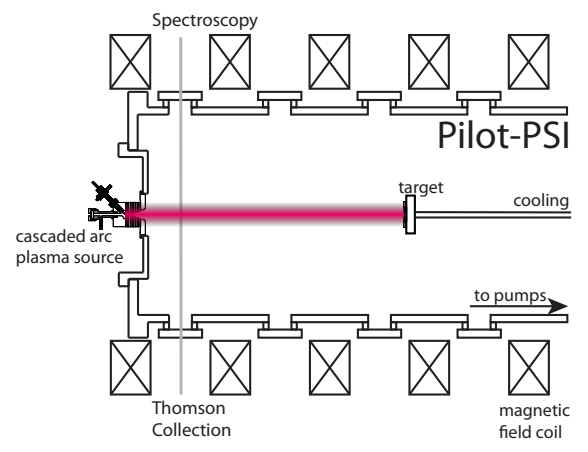

FIG. 1. Schematic of the Pilot-PSI setup. Optical Emission Spectroscopy and Thomson Scattering were performed at an axial distance of $5 \mathrm{~cm}$ from the cascaded arc source.

in which discharge currents ranging from 80 to 250 A were drawn between three tungsten cathodes and a copper-tungsten nozzle. Hydrogen gas flows are varied between 1 and 4 standard liters per minute. The plasma expands from a high pressure $\left(\sim 10^{4} \mathrm{~Pa}\right)$ in the cathode chamber to a low pressure $(\sim 2 \mathrm{~Pa})$ in the $1 \mathrm{~m}$ long, $0.4 \mathrm{~m}$ diameter vacuum vessel. The vessel pressure, monitored by a membrane gauge at the plasma source side of the vessel, is adjustable by variation of the rotation speed of its mechanical booster pump. A set of five field coils surrounding the vacuum vessel is used to generate magnetic fields of up to 1.6 T. More details of the experiment can be found elsewhere ${ }^{6}$.

Profiles of electron density and temperature are measured by means of Thomson Scattering at $5 \mathrm{~cm}$ downstream of the cascaded arc source anode ${ }^{27}$. Optical Emission Spectroscopy is performed using the in-house constructed high resolution spectrometer (HiRES), a singlepass spectrometer in Littrow configuration ${ }^{8}$. Light is collected at an axial distance of $5 \mathrm{~cm}$ from the plasma source, perpendicular to the magnetic field axis and at the same position as Thomson Scattering is performed. An array of 40 individual $0.4 \mathrm{~mm}$ diameter quartz fibers is used to relay the light to the HiRES spectrometer. In this way, a spectral range of approximately $4 \mathrm{~nm}$ can be investigated over the whole radius of the plasma jet for each discharge. The spatial resolution is $0.7 \mathrm{~mm}$. The measurements are line-of-sight integrated.

A relative intensity calibration was carried out using a USS-1200C integrating sphere. The instrument function was measured using an argon lamp. The instrument function is Gaussian shaped and has a 17 pm FWHM. Since the emission lines studied are also Gaussian shaped, the measured width is easily corrected for instrumental broadening using 


\begin{tabular}{rrrrrr}
\hline \hline State & $T_{e}$ & $\omega_{e}$ & $\omega_{e} x_{e}$ & $B_{e}$ & $\alpha_{e}$ \\
\hline$X^{1} \Sigma_{g}^{+}$ & 0 & 4401.21 & 121.33 & 60.853 & 3.062 \\
$a^{3} \Sigma_{g}^{+}$ & 95936 & 2664.83 & 71.65 & 34.216 & 1.671 \\
$d^{3} \Pi_{u}$ & 112700 & 2371.58 & 66.27 & 30.364 & 1.545 \\
\hline \hline
\end{tabular}

TABLE I. Molecular constants of hydrogen for electronic states of interest in $\mathrm{cm}^{-1}$ after the NIST database of Diatomic Molecules ${ }^{29}$.

the relation:

$$
\Delta \lambda_{\text {Doppler }}^{2}=\Delta \lambda_{\text {Measured }}^{2}-\Delta \lambda_{\text {Instrument }}^{2}
$$

\section{B. Vibrational temperatures from the Fulcher band}

The Fulcher- $\alpha$ band consists of the radiative transitions between the various vibrational

and rotational levels of the upper $d^{3} \Pi_{u}$ and lower $a^{3} \Sigma_{g}^{+}$electronic levels of the hydrogen molecule. Here, it is summarized how the vibrational and rotational temperature of the $\mathrm{X}^{1} \Sigma_{g}^{+}$electronic ground state are determined from the relative line intensities in the Fulcher $\alpha$ band.

Evaluation of the ground state vibrational temperature starts with calculation of the energy levels of the hydrogen molecule according to ${ }^{22}$ :

$$
G(\nu, J)=T_{e}+\omega_{e}\left(\nu+\frac{1}{2}\right)-\omega_{e} x_{e}\left(\nu+\frac{1}{2}\right)^{2}+B_{\nu} J(J+1)
$$

with

$$
B_{\nu}=B_{e}-\alpha_{e}\left(\nu+\frac{1}{2}\right)
$$

$\nu$ and $J$ are the rotational and vibrational quantum numbers, and the other symbols represent constants listed in Table I. Note that energy values are given in $\mathrm{cm}^{-1}$. It is assumed that the vibrational energy levels of the electronic ground state are populated according to a Boltzmann distribution, so that the distribution over the energy levels is known as function of $T_{v i b}$. The upper Fulcher state population is then calculated using the transition probabilities $^{28}$ or Franck-Condon factors ${ }^{20}$. Electron impact excitation is assumed as the dominant population mechanism of the upper Fulcher state. Because the excitation energy (approximately $14 \mathrm{eV}$ ) is large compared to the electron temperature, an additional factor 
TABLE II. Lines overlapping studied transitions after Crosswhite ${ }^{30}$.

\begin{tabular}{cccc}
\hline \hline Studied lines & \multicolumn{3}{c}{ Coinciding lines } \\
& Upper level Lower level & $\lambda(\mathrm{nm})$ \\
\hline & $i^{3} \Pi_{g}$ & $c^{3} \Pi_{u}$ & 603.1465 \\
& $H^{1} \Sigma_{g}^{+}$ & $B^{1} \Sigma_{u}^{+}$ & 603.1909 \\
$\mathrm{Q}_{(0-0)}(3)$ & $h^{3} \Sigma_{g}^{+}$ & $c^{3} \Pi_{u}$ & 603.1909 \\
& $g^{3} \Sigma_{g}^{+}$ & $c^{3} \Pi_{u}$ & 603.2171 \\
& - & $B^{1} \Sigma_{u}^{+}$ & 612.7246 \\
$\mathrm{Q}_{(1-1)}(2)$ & $d^{3} \Pi_{u}$ & $a^{3} \Sigma_{g}^{+}$ & 612.7648 \\
$\mathrm{Q}_{(1-1)}(3)$ & $g^{3} \Sigma_{g}^{+}$ & $c^{3} \Pi_{u}$ & 613.5136 \\
\hline \hline
\end{tabular}

is needed that accounts for the fraction of electrons with sufficient energy for excitation. Combining all these factors leads to the following expression for the upper Fulcher state population as function of $T_{v i b}$ and $T_{e}$ :

$$
f\left(\nu^{\prime}\right) \propto \sum_{\nu_{0}} q_{\nu^{\prime} \nu_{0}} e^{-\frac{E\left(\nu_{0}\right)}{k T_{v i b}}} e^{-\frac{\Delta E_{v i b}}{k T_{e}}}
$$

The measured intensities divided by the branching ratios ${ }^{20}$ are compared directly with the calculated population distributions. Varying $T_{v i b}$ in the calculation the sum of squares is minimized, which yields the vibrational temperature.

\section{Rotational temperatures from the Fulcher band}

The relative spectral line intensities within the Q-branch $(\Delta J=0)$ are used to determine the rotational temperature ${ }^{22}$. Some lines are disturbed by nearby lying lines, these lines cannot be used in the analysis, see Table II. The line intensity is converted to a relative population of the upper Fulcher state by taking into account the degeneracy of the upper state, and the transition strength. The relative population is then plotted on a logarithmic axis versus the energy of the ground state, given by Equation 6. If the upper level is populated according to a Boltzmann distribution, the points would show a linear dependence with slope $-\frac{E_{J}}{k T_{\text {rot }}}$.

This approach is only valid when the rotational distribution in the ground state follows a Boltzmann distribution. Energy exchange between the rotational and translational degrees 
of freedom takes place via inelastic molecule-molecule collisions, if these collisions dominate, the rotational temperature will be equal to the gas temperature ${ }^{31}$. Surface association of hydrogen molecules could lead to an overpopulation of higher rotational levels, but we assume that these processes are of minor influence, and that the lower levels are quickly thermalized. Absorption studies on similar plasmas confirm that the ground state lower rotational levels are Boltzmann populated with a temperature equal to ambient temperature ${ }^{10}$.

The ground state rotational distribution is assumed to be exactly imaged onto the excited state. No angular momentum is transferred during the electron impact excitation, and the excitation rate coefficients are independent of the rotational quantum number ${ }^{32,33}$.

Since the lifetime of the upper Fulcher state is about $31 \mathrm{~ns}$, and $\nu_{\text {coll }} / P \approx 6 \times 10^{4} \mathrm{~s}^{-1} \mathrm{~Pa}^{-1}$, only at pressures above $500 \mathrm{~Pa}$ collisional redistribution of the upper state population becomes important $^{34,35}$. At $500 \mathrm{~Pa}$, the time between collisions is equal to the radiative lifetime of the upper Fulcher state. Because of the low experimental pressures, redistribution can be neglected, i.e. the measured distribution reflects the ground state distribution ${ }^{14,15,36}$.

\section{Translational temperature determination}

The translational or gas temperature of the molecular hydrogen is determined from Doppler broadening of the emission line using the following formula:

$$
T_{\text {gas }}=\frac{m c^{2}}{8 k \ln 2}\left(\frac{\Delta \lambda_{F W H M}}{\lambda}\right)^{2}
$$

Since we operate at a comparatively low magnetic field of $0.4 \mathrm{~T}$, Zeeman splitting is small compared to the instrument function and can thus be neglected in the lineshape analysis ${ }^{18}$.

\section{RESULTS}

\section{A. Vibrational temperature and reaction rates}

The vibrational excitation of the Pilot-PSI plasma beam was evaluated under representative operational conditions: $3 \times 10^{20} \mathrm{~m}^{-3}$ and $3 \mathrm{eV}$ (radial profiles of density and temperature are plotted in Figure 2). The spectral regions containing the Q-branch of the first three vibrational transitions used for the analysis are plotted in Figure 3. It can be 


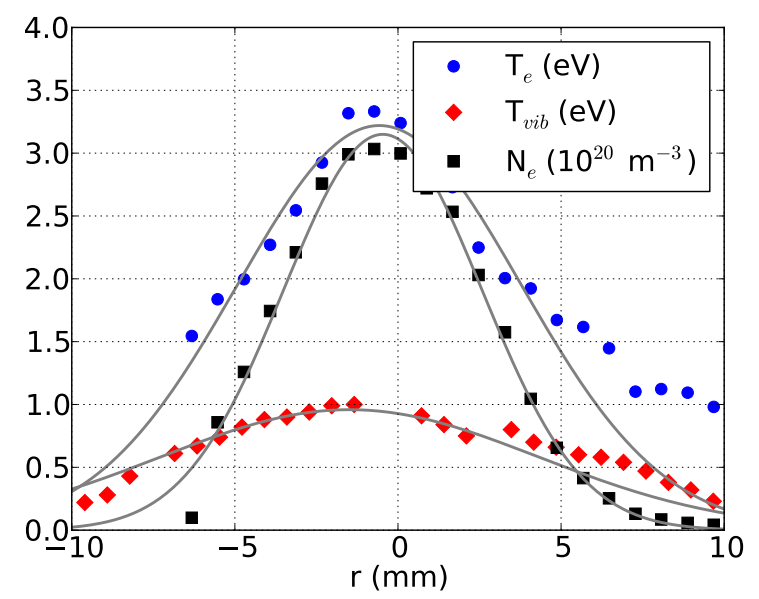

FIG. 2. Radial profiles of the electron temperature and density are measured by Thomson Scattering. Molecules diffusing into the plasma beam are heated to a vibrational temperature of $1 \mathrm{eV}$ over a path length of less than $1 \mathrm{~cm}$.

seen that this consists of four separate spectral regions, of which the width is determined by the spectral coverage of the spectrometer. The spectra were recorded in consecutive discharges, which limited the spectral region which could be studied in the available experimental time. Reproducibility was checked to be within $2 \%$. The rotational lines, which are annotated in the spectrum, show the 3:1 alternating intensity pattern caused by the hydrogen spin isomers. The combination of the Boltzmann distribution over the different states and the increasing degeneracy of higher lying states leads to the fact that e.g. the $\mathrm{Q}(0-0) 5$ peak is more intense than the $\mathrm{Q}(0-0) 1$ peak. There are however some peaks which do show an anomalous intensity, literature learns that these peaks are blended with other lines $^{30}$. Disturbed lines which are excluded in the data analysis are listed in Table II.

The Q1 line intensities were determined by fitting the line profiles with the Gauss function. An offset was allowed to account for continuum emission, which is significant at high densities studied here ${ }^{37}$. The first rotational peak in each vibrational branch was thus used to represent the population distribution in the upper Fulcher state. Vibrational temperatures in the ground state were obtained with the sum of squares minimization outlined in the previous section. This was repeated for each point along the vertical viewing cord covering the entire plasma beam diameter, yielding the vibrational temperature profile shown in Figure 2. The peak vibrational temperature of $1 \mathrm{eV}$ demonstrates that the molecules are 


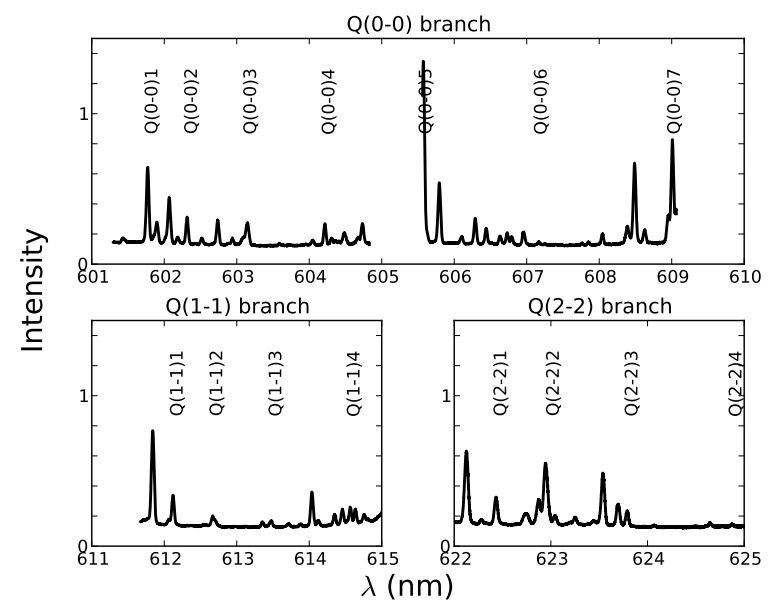

FIG. 3. Fulcher emission measured in the centre of the profiles shown in Figure 2. The vibrational temperature determined at this radial position is approximately $1 \mathrm{eV}$.

significantly heated by the plasma over a path length on the order of the plasma radius. At room temperature, hydrogen molecules travel this distance in microseconds, indicating that the heating takes place on this short timescale. Comparable vibrational temperatures of around $1 \mathrm{eV}$ have been observed earlier in tokamaks under comparable electron densities and temperatures using the same procedure ${ }^{38}$.

The $1 \mathrm{eV}$ vibrational temperature is used as input to calculate the reaction rates for negative-ion mediated MAR and ion-conversion MAR with the vibrationally resolved rates from Reiter et $a ._{.}{ }^{21}$. We note that a Boltzmann distribution is assumed also for the higher vibrational levels, although these were not directly probed ${ }^{39}$. At an electron temperature of $3 \mathrm{eV}$ and a vibrational temperature of $1 \mathrm{eV}$, the rate for negative-ion-mediated MAR is $2 \times 10^{-16} \mathrm{~m}^{3} \mathrm{~s}^{-1}$ and the rate for ion-conversion MAR is $1 \times 10^{-15} \mathrm{~m}^{3} \mathrm{~s}^{-1}$. Reaction rates for the two different MAR mechanisms fall within one order of magnitude thus both processes are of significance for plasma recombination under these conditions. Although these rates are greatly enhanced (an order of magnitude for the ion-conversion channel) compared to rates calculated without vibrational excitation, these are still not as high as assumed in previous experiments to explain the observed molecule depletion in the plasma beam of Pilot-PSI ${ }^{8}$. This hints at the importance of other mechanisms which also cause a hollow molecule density profile. 


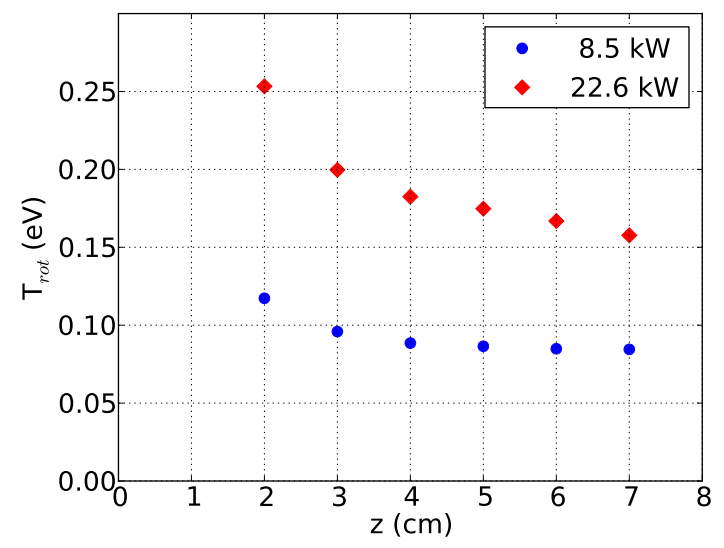

FIG. 4. The rotational temperature in a freely expanding plasma is close to the electron temperature $^{40}$ and cools in the expansion. The source was operating at 3 slm hydrogen with two different power inputs.

\section{B. Rotational and gas temperature determination}

Rotational temperatures were measured close to the plasma source exit when the plasma was freely expanding (i.e. zero magnetic field, all other results in this work were obtained in a magnetic field of $0.4 \mathrm{~T}$ ). These measurements are useful to connect the present experiments with existing literature, and served as a benchmark for the experiments under magnetized conditions. Results of axial scan at two different source input power levels are shown in Figure 4. Rotational temperatures of up to $0.25 \mathrm{eV}$ were found, which are significantly higher than rotational temperatures reported before in literature using a similar plasma source ${ }^{33}$. The measured rotational temperatures are close to the electron temperature measured by probes in expanding hydrogen plasmas ${ }^{40}$. The higher rotational temperatures are a consequence of operating at higher power: an energy input of around $2 \mathrm{eV}$ per molecule for the discharge with the highest rotational temperature was used. The rotational temperature cools in the expansion, as shown in Figure 4. The measurements in the free expansion are already an indication that we perform Fulcher spectroscopy under extreme conditions not previously explored.

The magnetic field confines the plasma into a narrow beam, which increases the electron density by orders of magnitude. The radial $\mathrm{H}_{2}$ emission profile also changes significantly when the magnetic field is switched on. The broad emission profile contracts to a more 


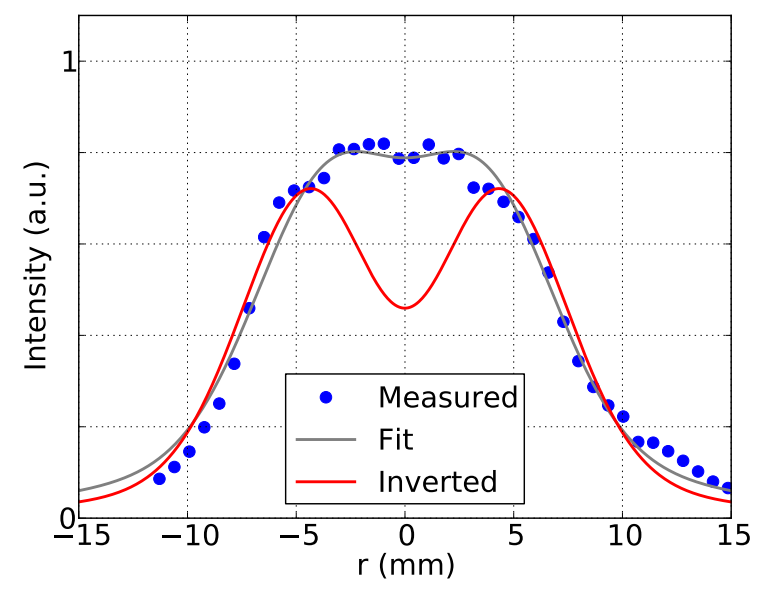

FIG. 5. The radial profile of the $\mathrm{Q}(0-0) 1$ intensity shown in Figure 6 for the magnetized plasma. Since the line-integrated measurement has a flat top profile, the Abel-inverted profile is hollow. This is a strong indication that the concentration of molecules is depleted towards the center of the plasma beam.



FIG. 6. Two spectra measured at a source current of $250 \mathrm{~A}$ and a hydrogen flow rate of $3 \mathrm{slm}$. In the magnetized plasma the hydrogen molecular spectrum changes: different $\mathrm{H}_{2}$ electronic states give rise to transitions overlapping with Fulcher peaks, and the relative line intensities within the Q-branch change. The $\mathrm{Q}(0-0) 3$ peak is comparatively intense because of overlap with other $\mathrm{H}_{2}$ emission lines, see Table II. 


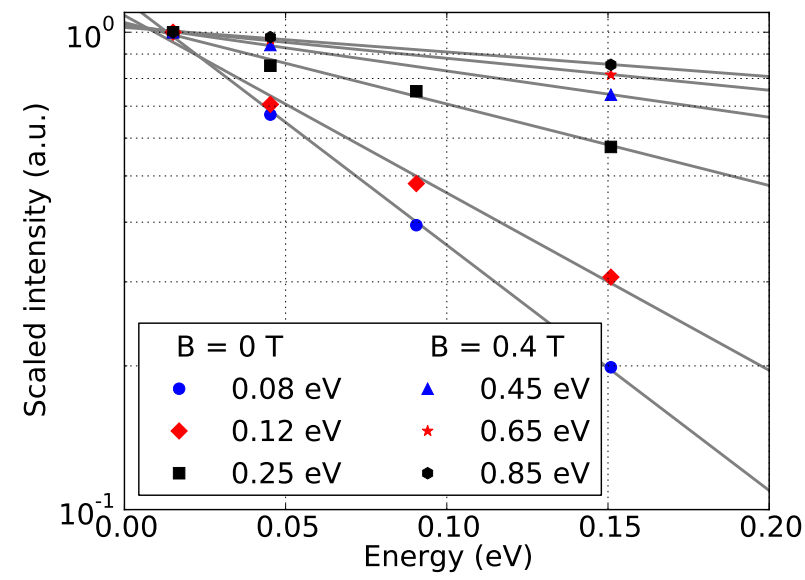

FIG. 7. Boltzmann plots for various plasma conditions, both freely expanding and magnetized. Note the absence of the $\mathrm{Q}(0-0) 3$ peak for the magnetized cases because of coinciding lines.

narrow, flat top profile. The measured emission is line integrated and hence a flat top profile corresponds to a hollow emission profile. As an illustration, Figure 5 shows a measured profile and the Abel-inverted profile. The inversion is performed by fitting Gaussians and using their invariance under Abel-inversion. The emission profile exhibits undoubtedly a minimum in the center. The exact value in the center, or the hollowness, is too sensitive to details in the inversion process to be quantified. A hollow emission profile means that the molecule density profile is hollow even more so because the plasma density and temperature profiles are peaked. However since the emission intensity depends non-linearly on the electron temperature and density, a quantitative statement on molecule depletion in the centre of the plasma beam is even harder to make.

The rotational line intensity distributions in the Fulcher emission change drastically when the magnetic field is switched on, as is seen in Figure 6: rotational excitation is strongly increased in the magnetized plasma compared to the freely expanding plasma, most likely due to shorter mean free paths at the increased plasma densities. For example, the $\mathrm{Q}_{(0-0)}(4)$ peak more than doubles in intensity while the height of the $\mathrm{Q}_{(0-0)}(1)$ peak stays approximately constant. This behavior is reflected in the Boltzmann plots shown in Figure 7. Indeed, rotational temperatures of up to $0.85 \mathrm{eV}$ are found.

In order to compare these high rotational temperatures with the actual gas temperature, we have evaluated the Doppler broadening of the rotational lines. The first line of the $\mathrm{Q}(0-0)$ 


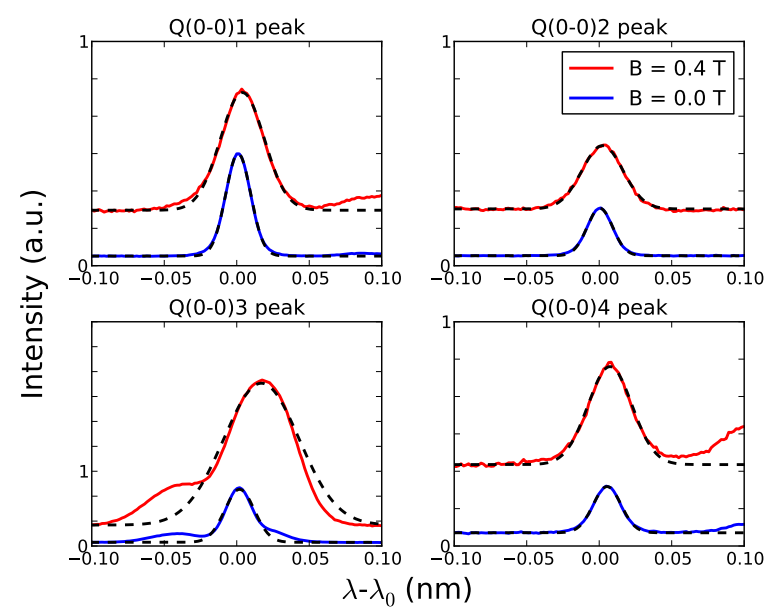

FIG. 8. This detail of Figure 6 shows the first four $\mathrm{Q}(0-0)$ peaks with Gaussian fits. The $\mathrm{Q}(0-0) 3$ peak is disturbed by nearby lying peaks and is not used in the rotational temperature determination. The broadening of the peaks for the magnetized case indicates a higher gas temperature.

and $\mathrm{Q}(1-1)$ branch was used to determine the translational (i.e. gas) temperature. Figure 8 shows the spectra of Figure 6 in more detail together with Gaussian fits to illustrate the broadening of the emission lines. Details of the line profile also confirm that the lines listed in Table II are a superposition of multiple lines. Within present work, these lines are simply disregarded, since the determination of the intensity of the overlapped lines was found to lead to ambiguous results.

Zeeman splitting as main cause of the line broadening was excluded by measuring a spectrum at $1.6 \mathrm{~T}$ and verifying that the line shape is still Gaussian, as shown in Figure 9. Since the Zeeman splitting scales with the field and with the inverse of the squared quantum number, and we see a negligible contribution at $1.6 \mathrm{~T}$ for the lowest rotational lines, we estimate that the Zeeman contribution at lower fields is less than ten percent ${ }^{18}$. It has therefore been neglected in the line shape analysis for the gas temperature determination.

Figure 10 shows a radial profile of the gas temperature and the rotational temperature. The rotational temperature is consistently lower than the translational temperature around the plasma center. We note that the rotational temperature data is only available near the plasma center as signal intensities become too low at the plasma edge for a stable Boltzmann fit.

Figure 11 shows the rotational temperature in the middle of the beam as function of the 


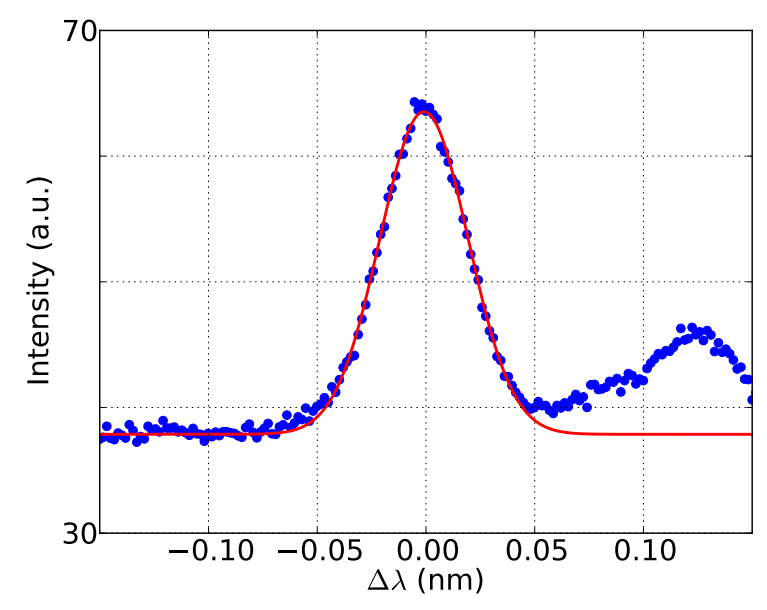

FIG. 9. The first $\mathrm{Q}(0-0)$ peak with a Gaussian fit at a field strength of $1.6 \mathrm{~T}$. At high fields the line shape stays Gaussian, this indicates that Zeeman splitting is not the main broadening mechanism. Comparing $\mathrm{Q}(0-0)$ lines with higher quantum numbers gives an upper bound to the amount of broadening expected at lower fields.

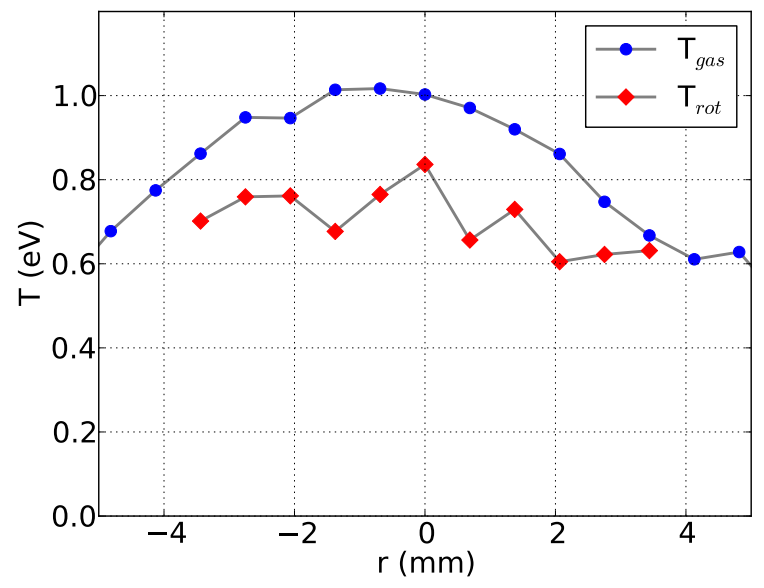

FIG. 10. A typical gas temperature and rotational temperature profile as measured in the magnetized plasma beam. The temperatures at radius zero were extracted from the spectrum shown in Figure 6.

gas temperature for a variety of discharges in order to get insight into the correspondence of the gas temperature and the rotational temperature. It demonstrates that the mismatch between the translational temperature and rotational temperature is a systematic effect. It is also clear that the $\mathrm{Q}(0-0)$ branch yields a higher rotational temperature than the $\mathrm{Q}(1$ - 




FIG. 11. Measured rotational temperatures shown as function of gas temperature for two vibrational branches. Each point represents one discharge. Lines are shown to guide the eye. Shots without magnetic field are denoted by a triangle.

1) branch. In literature a higher rotational temperature is often obtained from the lower vibrational branches ${ }^{16,17}$.

A possible explanation for the imbalance between the rotational and gas temperature is that there was not sufficient time for molecule-molecule collisions to equilibrate these different degrees of freedom at the time scale of heating ${ }^{31}$. This heating takes evidently place in the plasma by collisions with the hot plasma ions. Inspection of energy transfer cross sections for collisions between hot hydrogen ions and cooler molecules learns that the energy of the ions is preferentially transferred into the translational degrees of the hydrogen molecules ${ }^{35}$. As molecule densities are much lower than ion densities, the subsequent equilibration cannot take place during the time that the molecules reside in the plasma column. The consequence of this explanation is that the measured gas and rotational temperatures do not represent the residual gas properties throughout the vessel but only within the plasma column.

Additional support for this transient molecule heating by the plasma beam is the average rotation speed of the molecules. In Pilot-PSI, the ions rotate around the beam with velocities of a few $\mathrm{km} / \mathrm{s}$ because of $\mathbf{E} \times \mathbf{B}$ drift $^{40}$. This shows in spectra by the Doppler shift of the emission lines, which results from the velocity component of the emitter parallel to the spectroscopy observation chord. The molecules pick up some of this rotation, which is calculated from the Doppler shift of the molecular emission lines with the relation $v=$ 


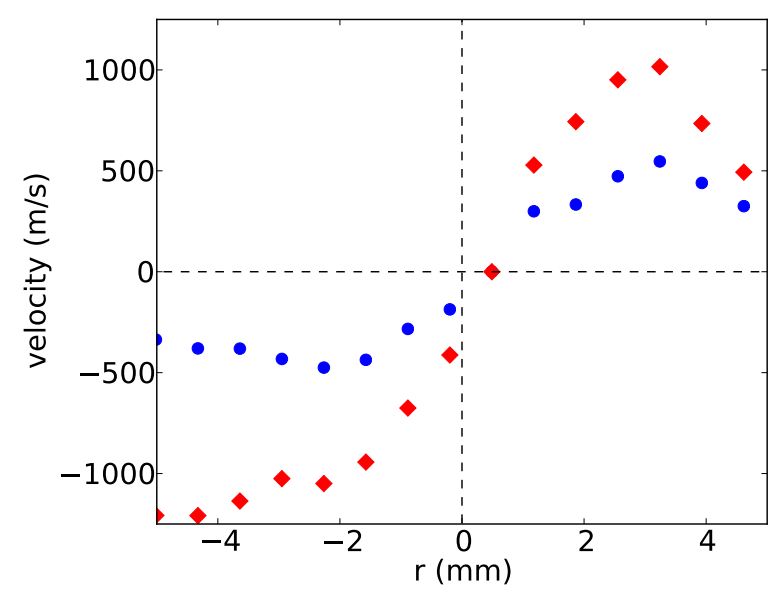

FIG. 12. Two molecular rotation profiles: Co-rotation of the molecules with the plasma is evidence for coupling between the molecules and plasma. Different plasma conditions show different molecule rotation speeds, 500 and $1100 \mathrm{~m} / \mathrm{s}$ for the discharges shown here.

$\left(\Delta \lambda / \lambda_{0}\right)$ c. Analysis of these Doppler shifts yields the velocity profiles shown in Figure 12. Note that the measured Doppler shifts are a few picometers, which is on the order of the linear dispersion of the spectrometer. The observed rotation speeds range from 500 to 1500 $\mathrm{m} / \mathrm{s}$, about an order of magnitude lower than the ion rotation velocity. This is yet another indication that the present molecular spectroscopy experiments probe the molecules at the same time scale as these are heated.

The consequence of the molecule heating inside the plasma column is rarefaction of the molecule density. According to the momentum balance, $n k T=$ constant, so the density of the molecules should decrease proportionally with the gas temperature increase. To estimate the importance of this effect, we constructed a model in which heating of the molecular influx by a stationary plasma column is evaluated. This simple model includes the following three terms in the power balance: the Maxwellian influx of cold molecules, the Maxwellian outflux of heated molecules and a term which describes the heating by the plasma. Dissociation of the molecules in the plasma is neglected for simplicity. The Maxwellian power flux is given by the area of the boundary region multiplied by the particle flux and the energy per particle. The heating term is given by the interaction volume multiplied with the amount of collisions per volume unit and the energy transfer per collision. The cross sections were taken from the work by Phelps et $a l .{ }^{35}$. The expressions for the terms in the power balance 
are thus:

$$
\begin{aligned}
& P_{\text {in }}=2 \pi R L \quad 1 / 4 n_{0} v_{0} \quad 2 k T_{0} \\
& P_{\text {out }}=2 \pi R L \quad 1 / 4 n_{b} v_{b} 2 k T_{b} \\
& P_{\text {heating }}=\pi R^{2} L n_{b} n_{e} \sigma v_{i} 3 k T_{i}\left(m_{i} / m_{n}\right)
\end{aligned}
$$

In order to estimate the rarefaction, the power balance $P_{\text {out }}=P_{\text {in }}+P_{\text {heating }}$ is solved for the density ratio between the cold gas outside the beam and the heated molecules inside the plasma:

$$
\frac{n_{0}}{n_{b}}=\frac{R n_{e} \sigma v_{i} 3 T_{i} m_{i}}{m_{0} v_{0} T_{0}\left(\sqrt{T_{b} / T_{0}}-1\right)}
$$

Taking a radius $R$ of $5 \mathrm{~mm}$, an electron density of $3 \times 10^{20} \mathrm{~m}^{-3}$, and assuming heating of the molecules to a temperature of $1 \mathrm{eV}$ by ions of $3 \mathrm{eV}$, the density ratio equals approximately 90. Although the model is very simple, it illustrates that heating alone can lead to a significant dilution of the molecules when comparing the centre of the plasma beam to the colder periphery.

A hollow $\mathrm{H}_{2}$ density profile under similar conditions was indirectly observed before by spectroscopy on atomic hydrogen ${ }^{8}$. Strongly enhanced rates for ion-conversion MAR were needed to explain the observed profiles. With this contribution we show that although the rates are significantly enhanced with respect to the lowest level, rarefaction because of heating is the dominant mechanism behind the observed hollow $\mathrm{H}_{2}$ density profiles.

\section{CONCLUSIONS}

$\mathrm{H}_{2}$ vibrational temperatures were determined from the Q-branch spectrum of the Fulcher$\alpha$ band. The vibrational population was used to compute effective reaction rates for negativeion-mediated MAR and ion-conversion MAR, which are significantly enhanced with respect to the rates for the lowest vibrational level.

$\mathrm{H}_{2}$ rotational temperatures were determined from the Q-branch spectrum of the Fulcher$\alpha$ band. In addition, translational temperatures were measured using line shape analysis. The translational temperature measured was systematically higher than the rotational temperature. This was explained by collisions with ions, since the cross section for momentum exchange is larger than for rotational excitation. The velocity profiles of the molecules 
were found to follow the ion velocity profile, giving a strong indication of coupling between molecules and plasma species. The main implication of the efficient heating of the molecules is rarefaction. Since from the momentum balance, $n k T$ is constant, the density of the molecules goes down proportionally with the temperature increase. This leads to a dilution of the molecules when comparing the centre of the plasma beam to the colder periphery. This effect, combined with the dissociation of the molecular influx, leads to a hollow density profile for the hydrogen molecules and thus causes a decreased effectiveness of molecular assisted processes.

These observations illustrate the general significance of rapid molecule heating in high density hydrogen plasma, e.g. in tokamak exhausts, for estimating molecular processes. Just taking residual gas properties on basis of e.g. wall temperatures and background pressure information is no longer justified. Furthermore, estimating gas temperatures on basis of rotational temperatures from Fulcher spectroscopy is compromised as both degrees of freedom may well be out of equilibrium.

\section{REFERENCES}

${ }^{1}$ D. Reiter, C. May, M. Baelmans, and P. Börner, Journal of Nuclear Materials 241243, 342 (1997).

${ }^{2}$ S. I. Krasheninnikov, A. Y. Pigarov, D. A. Knoll, B. LaBombard, B. Lipschultz, D. J. Sigmar, T. K. Soboleva, J. L. Terry, and F. Wising, Physics of Plasmas 4, 1638 (1997).

${ }^{3}$ K. Sawada and T. Fujimoto, Journal of Applied Physics 78, 2913 (1995).

${ }^{4}$ M. Bacal, A. M. Bruneteau, W. G. Graham, G. W. Hamilton, and M. Nachman, Journal of Applied Physics 52, 1247 (1981).

${ }^{5}$ C. Gorse, R. Celiberto, M. Cacciatore, A. Laganá, and M. Capitelli, Chemical Physics 161, 211 (1992).

${ }^{6}$ G. J. van Rooij, V. P. Veremiyenko, W. J. Goedheer, B. de Groot, A. W. Kleyn, P. H. M. Smeets, T. W. Versloot, D. G. Whyte, R. Engeln, D. C. Schram, and N. J. L. Cardozo, Applied Physics Letters 90, 121501 (2007).

${ }^{7}$ M. de Graaf, R. Severens, R. Dahiya, M. C. M. van de Sanden, and D. C. Schram, Phys. Rev. E 48, 2098 (1993).

${ }^{8}$ A. E. Shumack, V. P. Veremiyenko, D. C. Schram, H. J. de Blank, W. J. Goedheer, H. J. 
van der Meiden, W. A. J. Vijvers, J. Westerhout, N. J. L. Cardozo, and G. J. van Rooij, Physical Review E 78, 046405 (2008).

${ }^{9}$ R. K. Janev, Physica Scripta 2002, 94 (2002).

${ }^{10}$ P. Vankan, D. C. Schram, and R. Engeln, Chemical Physics Letters 400, 196 (2004).

${ }^{11}$ R. F. G. Meulenbroeks, R. A. H. Engeln, J. A. M. van der Mullen, and D. C. Schram, Physical Review E 53, 5207 (1996).

${ }^{12}$ J. H. M. Bonnie, P. J. Eenshuistra, and H. J. Hopman, Physical Review A 37, 1121 (1988).

${ }^{13}$ G. C. Stutzin, A. T. Young, A. S. Schlachter, K. N. Leung, and W. B. Kunkel, Chemical Physics Letters 155, 475 (1989).

${ }^{14}$ L. Tomasini, A. Rousseau, G. Gousset, and P. Leprince, Journal of Physics D: Applied Physics 29, 1006 (1996).

${ }^{15}$ S. I. Gritsinin, I. A. Kossyi, N. I. Malykh, V. G. Ral'chenko, K. F. Sergeichev, V. P. Silakov, I. A. Sychev, N. M. Tarasova, and A. V. Chebotarev, Journal of Physics D: Applied Physics 31, 2942 (1998).

${ }^{16}$ B. Heger, U. Fantz, and K. Behringer, Journal of Nuclear Materials 290-293, 413 (2001).

${ }^{17}$ U. Fantz, Contributions to Plasma Physics 42, 675 (2002).

${ }^{18}$ T. Shikama, S. Kado, H. Zushi, and S. Tanaka, Physics of Plasmas 14 (2007).

${ }^{19}$ G. S. Fulcher, Astrophysical Journal 37, 60 (1913).

${ }^{20}$ U. Fantz and B. Heger, Plasma Physics and Controlled Fusion 40, 2023 (1998).

${ }^{21}$ D. Reiter, The data file H2VIBR: Additional Molecular Data for EIRENE: vibrationally resolved H2 (2005).

${ }^{22} \mathrm{G}$. Herzberg, Molecular spectra and molecular structure, Vol. I. (Krieger publishing company, 1989).

${ }^{23}$ I. P. E. G. on Divertor, I. P. E. G. on Divertor Modelling, Database, and I. P. B. Editors, Nuclear Fusion 39, 2391 (1999).

${ }^{24}$ R. A. Pitts, A. Kukushkin, A. Loarte, A. Martin, M. Merola, C. E. Kessel, V. Komarov, and M. Shimada, Physica Scripta 2009, 014001 (2009).

${ }^{25}$ A. E. Shumack, D. C. Schram, J. Biesheuvel, W. J. Goedheer, and G. J. van Rooij, Phys. Rev. E 83, 036402 (2011).

${ }^{26}$ G. M. W. Kroesen, D. C. Schram, and J. C. M. de Haas, Plasma Chemistry and Plasma Processing 10, 531 (1990). 
${ }^{27}$ H. J. van der Meiden, R. S. Al, C. J. Barth, A. J. H. Donne, R. Engeln, W. J. Goedheer, B. de Groot, A. W. Kleyn, W. R. Koppers, N. J. L. Cardozo, M. J. van de Pol, P. R. Prins, D. C. Schram, A. E. Shumack, P. H. M. Smeets, W. A. J. Vijvers, J. Westerhout, G. M. Wright, and G. J. van Rooij, Review of Scientific Instruments 79, 013505 (2008). ${ }^{28}$ U. Fantz and D. Wünderlich, Atomic Data and Nuclear Data Tables 92, 853 (2006). ${ }^{29}$ NIST, "Database of Diatomic Molecules: Hydrogen," http://webbook.nist.gov/cgi/ cbook. cgi?ID=C1333740\&Units=SI\&Mask=1E9F (1976), [Online; accessed 27-July-2011].

${ }^{30} \mathrm{H}$. M. Crosswhite, The hydrogen molecule wavelength tables of Gerhard Heinrich Dieke (Wiley-Interscience, 1972).

${ }^{31}$ S. A. Astashkevich, M. Käning, E. Käning, N. V. Kokina, B. P. Lavrov, A. Ohl, and J. Röpcke, Journal of Quantitative Spectroscopy and Radiative Transfer 56, 725 (1996).

${ }^{32}$ B. P. Lavrov, V. N. Ostrosky, and V. I. Ustimov, Journal of Physics B: Atomic and Molecular Physics 14, 4389 (1981).

${ }^{33}$ M. J. de Graaf, A new hydrogen particle source, Ph.D. thesis, Technical University Eindhoven (1994).

${ }^{34}$ S. Astashkevich and B. Lavrov, Optics and Spectroscopy 92, 818 (2002).

${ }^{35}$ A. V. Phelps, Journal of Physical Chemical Reference Data 19, 653 (1990).

${ }^{36}$ R. K. Garg, T. N. Anderson, R. P. Lucht, T. S. Fisher, and J. P. Gore, Journal of Physics D: Applied Physics 41, 095206 (2008).

${ }^{37}$ R. F. G. Meulenbroeks, M. F. M. Steenbakkers, Z. Qing, M. C. M. van de Sanden, and D. C. Schram, Phys. Rev. E 49, 2272 (1994).

${ }^{38}$ U. Fantz, K. Behringer, J. Gafert, D. Coster, and A. U. Team, Journal of Nuclear Materials 266269, 490 (1999).

${ }^{39}$ M. Capitelli, R. Celiberto, G. Colonna, G. D’Ammando, O. D. Pascale, P. Diomede, F. Esposito, C. Gorse, A. Laricchiuta, S. Longo, and L. D. Pietanza, Journal of Physics B: Atomic, Molecular and Optical Physics 43, 144025 (2010).

${ }^{40}$ V. P. Veremiyenko, An ITER-relevant magnetized hydrogen plasma jet, Ph.D. thesis, Eindhoven University of Technology, Eindhoven, Netherlands (2006). 